\title{
LA VULNERABILIDAD DESDE LA PSICOLOGÍA ORGANIZACIONAL
}

\author{
Andrés Muñoz Alvis ${ }^{1}$ \\ Oscar Iván Gutiérrez Carvajal ${ }^{2}$
}

\section{Resumen}

Las organizaciones se constituyen como grupos sociales estructurados, en donde se presentan situaciones de vulnerabilidad para las personas que las conforman. Algunas formas de vulnerabilidad son sistémicas a la organización. Se generan por las dinámicas propias en las relaciones jerárquicas estipuladas en su organigrama, que determinan las líneas de poder y, en parte, las formas en que las personas se relacionan. Otras, en cambio, están asociadas a los estilos de personalidad y dirección de sus miembros, quienes, con o sin intención, ejercen conductas que vulneran el bienestar de los otros. El objetivo del documento es ilustrar al lector sobre las formas en que se presenta la vulnerabilidad en las organizaciones y, a su vez, indicar algunas estrategias que permitan la reducción de los niveles de vulnerabilidad en las organizaciones. Pues, si se pasa por alto este fenómeno, se favorecerá la aparición de síntomas como el burnout, la ansiedad, el humor depresivo y las enfermedades del tracto digestivo, entre otras, que perjudican al empleado, a su grupo familiar y social, dentro y fuera de la organización. Como conclusión, en el documento se muestran las múltiples dimensiones desde las que se presenta la vulnerabilidad en las organizaciones, pero, también, aborda al ser humano como sujeto orgánico, cuya salud se ve afectada o beneficiada

1 Universidad del Norte, Colombia, Kilómetro 5 Antigua vía a Puerto Colombia, Departamento de Psicología. Teléfono: 3008027818. aalvis@uninorte.edu.co. Doctorando en Psicología de Las Organizaciones, Magíster en Desarrollo Organizacional. Especialista en Psicología del Consumidor. Área de conocimiento: Psicología en la Organizaciones y Desarrollo Organizacional. Grupo de Investigación: Psicología Universidad del Norte. Línea: Organización y gestión del talento Humano.

2 Universidad del Norte, Colombia, Kilómetro 5 Antigua vía a Puerto Colombia, Departamento de Psicología. Teléfono: 3176807597. oscar.ivan.gutierrez@gmail.com. Doctorando en Psicología de Las Organizaciones. Magister en Administración de Instituciones Educativas. Área de conocimiento: Evaluación de desempeño, bienestar, psicometría y evaluación. BECARIO COLCIENCIAS. 
por la organización donde trabaja, en la que pasa al menos dos tercios de su día a día.

Palabras clave: vulnerabilidad, psicología organizacional, salud mental, bienestar

\section{Abstract}

The organizations are constituted as structured social groups, where situations of vulnerability are presented to the people who make them up. Some forms of vulnerability are systemic to the organization. They are generated by their own dynamics in the hierarchical relationships stipulated in their organization chart, which determine the lines of power and, in part, the ways in which people relate to each other. Others, on the other hand, are associated with the personality styles and direction of their members, who, with or without intention, exercise behaviors that violate the welfare of others. The objective of the document is to illustrate to the reader about the ways in which vulnerability is presented in organizations and, in turn, to indicate some strategies that allow the reduction of levels of vulnerability in organizations. Well, if this phenomenon is overlooked, the appearance of symptoms such as burnout, anxiety, depressive mood and diseases of the digestive tract, among others, which harm the employee, their family and social group, will be encouraged. outside the organization. In conclusion, the document shows the multiple dimensions from which vulnerability is presented in organizations, but also addresses the human being as an organic subject, whose health is affected or benefited by the organization where he works, in which He spends at least two thirds of his day to day.

Key words: vulnerability, organizational psychology, mental health, well-being 


\section{Introducción}

La vulnerabilidad en el contexto del trabajo y de las organizaciones es vista dentro de los factores ambientales o personales, que pueden poner en riesgo la seguridad, y la salud física y mental del trabajador. El trabajador es vulnerable debido a la exposición a ciertos factores y, a su vez, la salud y la seguridad de los trabajadores puede afectar a la organización. No obstante, aunque todo trabajador es vulnerable, aquellos trabajos que implican alta explotación hacen que se sea más vulnerable que otros.

La Organización Mundial de la Salud (OMS) indica, en su reporte sobre Entornos Laborales Saludables del año 2010 que, dentro de los grupos de trabajadores que son altamente explotados, y por tanto más vulnerables, se encuentran los niños, las mujeres, los inmigrantes, las personas sin hogar y también quienes realizan trabajos forzados o eventuales. Asimismo, las mujeres presentan mayor tendencia a convertirse en víctimas de acoso sexual en el espacio de trabajo, por lo que este grupo, en particular, es mucho más vulnerable que otros.

De igual forma, dicho reporte afirma que el costo humano en el trabajo es incalculable. Dentro de sus cifras explica que, solo en 1998, aproximadamente 346.000 de los decesos por enfermedades y accidentes se dieron en el espacio de trabajo, y las cifras continúan en aumento. También, informa que dentro de las enfermedades contagiosas y no contagiosas, relacionadas con el trabajo, se encuentran el VIH, la hepatitis B y C, varias formas de cáncer, enfermedades respiratorias crónicas, enfermedades de la piel, desórdenes físico-neurológicos, problemas reproductivos como la infertilidad y abortos, el humor depresivo, la ansiedad, el burnout, y muchos otros (OMS, 2010).

Este escenario nos muestra que el mundo del trabajo - que es tan valioso para las personas y las sociedades- puede tener consecuencias en la salud, entendida esta como un estado de completo bienestar físico, mental y social, y no la simple ausencia de la enfermedad (OMS, 2010). En este punto, es de aclarar que la definición de la OMS integra una visión clásica de la salud como ausencia de enfermedad, y también una visión más reciente orientada al bienestar. Este último, el bienestar, ha sido comprendido, gracias a las investigaciones recientes, como un estado 
multidimensional de bienestar psicológico, bienestar subjetivo y bienestar social (Keyes, 2005).

La gestión de la salud y el bienestar en el trabajo es un asunto complejo debido a que existen múltiples condiciones del trabajo, de la organización y del individuo, que conviven como un sistema en el que las condiciones físicas y mentales interactúan, protegiendo o poniendo en riesgo a los trabajadores. Un ejemplo de dicha interacción es la explicación del Modelo de Accidente Traumático del Instituto Americano del Estrés, donde el estrés puede alterar el desempeño generando errores, y este llevar a accidentes con consecuencias de daños físicos Kamp (1994). En esta lógica, algunos factores físicos pueden generar afectaciones psicológicas o viceversa.

Actualmente, se evidencia un crecimiento en el interés por la salud física y mental en el trabajo y, con ello, los entornos de trabajo más saludables. Para la OMS (2010), un entorno de trabajo saludable es aquel en el que los trabajadores y jefes colaboran en un proceso de mejora continua para promover y proteger la salud, la seguridad y el bienestar de los trabajadores, y la sustentabilidad del ambiente de trabajo con base en los siguientes indicadores: (a) la salud y la seguridad, concernientes al espacio físico de trabajo; (b) la salud, la seguridad y el bienestar, concernientes al medio psicosocial del trabajo, incluyendo la organización del mismo y la cultura del ambiente de trabajo; (c) los recursos de salud personales en el espacio de trabajo; y (d) las formas en que la comunidad busca mejorar la salud de los trabajadores, la de sus familias y la de otros miembros de la comunidad.

Un área de la psicología encargada de trabajar por la disminución de la vulnerabilidad en el trabajo es la psicología de la salud ocupacional. Esta área tiene como finalidades: (a) crear ambientes de trabajo seguros y saludables, para promover organizaciones y personas saludables; y (b) ayudar a las organizaciones a desarrollar sistemas inmunes, que metabolicen las toxinas emocionales y psicosociales que afectan el ambiente de trabajo. Para lograrlo, la psicología de la salud en el trabajo, desarrolla intervenciones a nivel primario y se hacen en tres niveles: individual, grupal y organizacional (Gómez, 2009). De igual manera, la psicología positiva es un área que aporta en el campo de la salud, centrándose en los recursos de los trabajadores que facilitan su bienestar, haciendo énfasis en el fortalecimiento el desarrollo de las potencialidades humanas. 


\section{Factores psicosociales que representan riesgos para la salud de los trabajadores}

Algunos riesgos psicosociales que pueden poner en riesgo la salud mental y física del trabajador son el estrés, la percepción de sobrecarga laboral, la alta demanda y el bajo control, los conflictos en el rol, la ambigüedad de las tareas, el trabajo nocturno, las horas extras, el liderazgo de pobre calidad, la agresión en el espacio de trabajo y el conflicto trabajofamilia. Dentro de los riesgos físicos se encuentran los riesgos mecánicos y eléctricos; los deslizamientos y las caídas de alturas; los riesgos ergonómicos, como movimientos repetitivos, posiciones forzadas y esfuerzo excesivo; o los riesgos de accidentes en vehículos (Leka \& Cox, 2009). A continuación, se comentan algunos de dichos factores.

\section{El contenido de la tarea}

Existen trabajos cuyas tareas pueden resultar más o menos exigentes para las habilidades y recursos del trabajador. En ambos casos, esto puede resultar un factor de vulnerabilidad. La variedad de la tarea, la complejidad de las mismas y la suficiencia de información sobre las actividades son aspectos relacionados con la salud. Un ejemplo de esto es el estudio desarrollado por Donders, Roskes, y van der Gulden (2007) con 1.791 trabajadores, quienes encontraron que aspectos del trabajo, como la variedad de las tareas y la buena información, han resultado relacionadas con menor fatiga en el trabajo, menor agotamiento emocional y con mayor satisfacción.

En el mismo sentido, Palumbo et al. (2017), llevaron a cabo un estudio con 91.627 mujeres y sus análisis reportaron que los trabajos que requieren resolución de problemas complejos, aprendizaje activo y pensamiento crítico, se asocian con un mejor funcionamiento físico. Asimismo, los resultados indican que aquellos trabajos que requieren de alto rendimiento físico podrían rediseñarse, incluyendo variedad con algunas actividades relacionadas con problemas complejos, para reducir problemas de salud en el largo plazo. 


\section{Carga y ritmo de trabajo}

En la misma línea, si las características de la tarea superan las capacidades o recursos cognitivos de las personas, esto puede generar sobrecarga mental; si superan los recursos emocionales, pueden generar sobrecarga emocional; o, si se trata de recursos músculo-esqueléticos, se puede generar una sobrecarga física. Existen, al menos, tres tipos de sobrecarga laboral: (a) cuantitativa, es decir, demasiado trabajo; (b) cualitativa, que se refiere al trabajo que es demasiado difícil para una persona; y (c) subcarga, una situación donde el individuo no se siente suficientemente desafiado por el trabajo (Arnold et al., 2012).

De igual manera, trabajar a un ritmo rápido, de forma intensiva y tener que cumplir con fechas límite, con frecuencia son factores que hacen vulnerables a los trabajadores a padecer de estrés (Arnold et al., 2012). Diferentes estudios han demostrado cómo la sobrecarga de trabajo puede afectar la salud y el bienestar. Por ejemplo, González y Gutiérrez (2006) determinaron que la duración de la jornada, la demanda mental, la demanda temporal y la frustración ante la tarea, pueden considerarse factores de riesgo determinantes para el estrés laboral de los trabajadores.

Asimismo, Rydstedt, Johansson y Evans (1998) identificaron que los cambios en la carga de trabajo, durante un período de 18 meses, se asociaron fuertemente con el esfuerzo percibido para llevar a cabo el trabajo, así como también con el derrame de fatiga desde el trabajo hasta el tiempo de ocio y la vida hogareña. También, determinaron que el aumento de la carga de trabajo se asoció con un mayor agotamiento después del trabajo, dificultades para relajarse después del trabajo, problemas para hacer frente a las demandas en el hogar y el uso recreativo del tiempo libre; como con síntomas psicosomáticos, pero no con el uso de sustancias farmacológicas potencialmente relacionadas con el estrés.

\section{Horario de trabajo}

El trabajo por turnos, por ejemplo, puede afectar negativamente la salud, la seguridad o la capacidad de las personas para desempeñarse en la manera adecuada. Los horarios pueden tener efectos como cambios en los ciclos de sueño, trastornos emocionales, problemas digestivos, enfermedades del corazón, entre otras. Rodríguez y Méndez (2016) encontraron en una 
investigación que el síndrome de Burnout prevalece de forma significativa en los turnos nocturnos y especiales, y, además, este genera mayor riesgo de cansancio emocional y despersonalización en los empleados. También se encontró, por ejemplo, relación entre los turnos de trabajo y el aumento del índice de masa corporal (Buden et al., 2016).

Fernández y Piñol (2000) explican que los turnos de trabajo tienen efectos negativos sobre la salud. Según estos autores, las investigaciones llevadas a cabo en los últimos años muestran la presencia de numerosas repercusiones negativas sobre la salud de los trabajadores afectados. Igualmente, indican que algunos horarios pueden generar efectos adversos que conllevan a trastornos del sueño, síndrome de fatiga crónica, estrés laboral, sintomatología depresiva y diversos problemas familiares y sociales. Asimismo, quienes trabajan en horarios fuera de la fisiología normal del ciclo del sol, presentan mayor absentismo, menor rendimiento y accidentes de trabajo, en comparación con los trabajadores con un horario convencional.

\section{Control y autonomía}

El control en el trabajo es otro factor de vulnerabilidad usualmente referido al estrés. La investigación indica que la falta de influencia en la forma en que el trabajo se organiza y se desempeña puede ser perjudicial para el bienestar psicológico y físico (Arnold et al., 2012). Según indican Bond, Flaxman y Loivette (2006), la investigación con respecto al control muestra que los niveles más altos de control pronostican longitudinalmente mejores medidas objetivas de desempeño y calificaciones de desempeño, así como también niveles más bajos de ausentismo e intención de rotación.

De igual forma, la autonomía con respecto a la posibilidad de tomar decisiones en el trabajo se ha relacionado con la satisfacción de la vida (Polo, Fernández \& Ramírez, 2012), con engagement (Polo, 2012), otros aspectos de la salud mental (Park \& Searcy, 2012) y con satisfacción laboral (Morgeson \& Campion, 2002). Así las cosas, el tener control y autonomía en el trabajo juega a favor de la salud de los empleados facilitando su desempeño. 


\section{Intimidación y Moobing}

Otra forma de vulnerar los derechos de un trabajador es mediante el acoso laboral, que se refiere al maltrato que uno o varios miembros de la empresa ejerzan sobre uno o varios empleados, sin importar en ello el rango o cargo que el acosador o acosado tenga en la estructura organizacional. De acuerdo con la ley 10 de 2006, en Colombia se define el acoso laboral como:

... toda conducta persistente y demostrable, que ejercida sobre un empleado, trabajador por parte de un empleador, un jefe o superior jerárquico inmediato o mediato, un compañero de trabajo o un subalterno, encaminada a infundir miedo, intimidación, terror y angustia, a causar perjuicio laboral, generar desmotivación en el trabajo o inducir la renuncia del mismo. (Congreso de la República de Colombia, 2018)

Por su parte, (Arnold et al., 2012, p. 589) asocia el maltrato con el acoso, al cual define como "Actos negativos hacia una persona, donde el perpetrador tiene más poder que la víctima". Un nuevo concepto para denominar el maltrato o el acoso es el denominado moobing, al que Topa, Depolo y Morales (2007, p. 88) refieren como "un proceso de agresión sistemática y repetida por parte de una persona o grupo hacia un compañero, subordinado o superior." Por su parte (Peralta, 2004) lo define como una "conducta insultante, maliciosa, ofensiva, intimidatoria, abusiva del poder y persistente, que hace sentir a la persona burlada, humillada o vulnerable y que socava su auto estima y le causa ansiedad".

A pesar de las definiciones que se puedan hallar sobre el acoso laboral o moobing, una dificultad frecuente para el psicólogo de las organizaciones, o para cualquier actor involucrado en la el acoso laboral, es identificar cuándo realmente existe. Esto, debido a que en la mayoría de las ocasiones este se presenta de manera implícita en las relaciones laborales; en muchas ocasiones ni siquiera existe la intención de causar acoso. Incluso, algunas normas subjetivas de la organización pudieran ser vistas por algunos como acoso, por ejemplo, algunos jefes corrigen a sus subordinados mediante expresiones que pudiesen ser violentas, pero, no por ello, desean ser violentos, simplemente pueden considerarse personas de carácter fuerte, sin percatarse de que algunos tratos limitan con el maltrato y el acoso laboral. 
A partir de lo anterior, es importante que los psicólogos que laboren para una empresa tengan presente los posibles comportamientos asociados al acoso laboral, no solo porque su trabajo implica aumentar el bienestar de las personas, sino porque las personas que son víctimas de estos comportamientos reducen sus percepciones de satisfacción laboral y, con ello, se afectan otras variables relacionadas con el desempeño, la motivación y el sentido de pertenencia, entre otros. De hecho, Muñoz, López y González (2018) realizaron una investigación en la que hallaron que el moobing y la satisfacción laboral mantienen una relación causal. En este sentido el moobing genera insatisfacción laboral y, de allí, se desprenden otras percepciones y conductas negativas para la persona y para la organización.

Para identificar cuando hay acoso laboral, la Ley 1010 de 2006 Congreso de Colombia, (2006) propone una serie de formas de maltrato que el psicólogo de las organizaciones debería conocer, toda vez que se constituye en el argumento jurídico para identificar si existe acoso laboral en un contexto determinado, así, el acoso laboral se puede presentar bajo las siguientes formas:

- Maltrato Laboral: Implica todas las formas de agresión física o psicológica que se ejerza sobre un empleado o un grupo de ellos, de tal manera que se afecte el buen nombre, la integridad, los derechos de las personas e incluso su autoestima.

- Persecución Laboral: Se relaciona con todas las acciones reiteradas que permiten inferir la intención de conducir al empleado a presentar su renuncia. Acciones como sobrecargar de actividades al empleado, y cambiar frecuente y repentinamente su horario de trabajo sin justificación racional pueden indicar un caso de persecución laboral.

- Discriminación Laboral: Se refiere a cualquier discriminación que se haga sobre un empleado por su condición racial, religiosa, socio-económica, de preferencia sexual, nacionalidad, origen familiar y cualquier otra condición que implique alguna forma de segregación del empleado.

- Entorpecimiento Laboral: Se relaciona con las conductas asociadas a obstaculizar el trabajo de un trabajador, ocultando 
información, insumos, documentos y demás aspectos necesarios para su adecuado desempeño en la organización.

- Inequidad laboral: Resulta de la distribución inequitativa de las funciones o de los beneficios laborales, generando, en el primer caso, una sobrecarga de funciones sobre uno o varios empleados y un exceso de beneficios sobre uno o varios empleados. Es importante tener en cuenta que la existencia de inequidad laboral está sujeta a que las diferencias a las que se hace referencia deben carecer de argumentos suficientes que las justifiquen.

- Desprotección Laboral: Implica omitir las condiciones mínimas de seguridad y coberturas legales que propendan por el cuidado de la salud del empleado.

El acoso laboral puede generarse sobre una persona o sobre un grupo de personas. En el primer caso, considere un jefe que acosa laboralmente a su asistente; en el segundo considere a un jefe de operaciones que acosa laboralmente a su equipo de trabajo; e, incluso, podría darse el caso de algunas personas generando acoso laboral sobre otro grupo.

En algunos casos, el acoso laboral puede originarse a partir de procesos inadecuados de selección de personal, en los que se termina contratando a una persona con características personales proclives a cualquier forma de acoso laboral. En otras ocasiones, estructuras organizacionales mal diseñadas terminan creando el escenario propicio para la aparición de este fenómeno; por ejemplo, la falta de diseños de puesto, de manuales de funciones, que permiten que, "de manera clara las funciones, responsabilidades, conocimientos, experiencias y competencias que debe poseer cada responsable del puesto" (Sarango, 2017, p. 2), pueden generar situaciones de acoso originadas por deficiencias en la estructura de la empresa. Esto se da porque no existe claridad sobre el rol y las responsabilidades de cada empleado en la organización.

Es necesario tener en cuenta que, a pesar de que el acoso es un fenómeno colectivo, que puede involucrar no solo a varias personas, sino a varios grupos. Sin embargo, bien sea por acoso individual o colectivo, las personas acosadoras o maltratadoras laboralmente presentan rasgos comunes: suelen ser irritables, suspicaces, con baja capacidad de complacencia ante un estímulo y, por lo general, tienen bajo control de 
sus emociones y sus impulsos (Camargo \& Puentes, 2010). No obstante, otros factores de diversa índole también pueden influir en la generación de conductas asociadas al acoso o maltrato laboral.

Cualquier trabajador que perciba que es acosado laboralmente, tendrá, entre sus derechos, el de acudir a una dependencia del Ministerio del Trabajo y reportar tal situación. Sin embargo, deberá poder diferenciar cuándo es o no víctima de acoso. Por ejemplo, los llamados de atención ante un trabajo desempeñado de forma ineficiente no podrán ser considerados como acoso laboral; la exigencia del cumplimiento de las actividades estipuladas en el manual de funciones, tampoco puede ser considerada como acoso, salvo que las mismas sobrepasen material y físicamente las capacidades de "cualquier" persona para desarrollarlas. Tampoco se entenderán como acoso las exigencias razonables de fidelidad y lealtad laboral: la solicitud de apoyar en labores extras cuando se presentan condiciones difíciles en la organización (Congreso de la República de Colombia, 2006).

Finalmente, en las organizaciones donde prevalecen situaciones de acoso laboral, la calidad de vida de las personas suele ser menor y, habitualmente, se presenta una baja tasa de satisfacción laboral, situación contraproducente para la organización, ya que un empleado satisfecho tendrá mayores probabilidades de alcanzar un mejor desempeño en sus actividades y logrará una mayor integración social. El siguiente apartado aborda precisamente la calidad de vida y el papel que juega la satisfacción laboral en el alcance de esta.

\section{Calidad de vida en el trabajo y satisfacción laboral}

La calidad de la vida siempre ha sido un tema de amplias discusiones desde diversas perspectivas teóricas y disciplinares. Una de estas perspectivas es la psicología y, en este caso, la psicología aplicada al contexto de las organizaciones, dado que las personas suelen pasar gran parte de su vida adulta en sus lugares de trabajo, en donde establecen relaciones con otros, asumen roles sociales, adquieren competencias y obtienen el sustento para sus familias. Por lo que es esperable que la satisfacción laboral percibida incida directamente sobre su calidad de vida.

La definición de satisfacción laboral ha sido un amplio tema de debate, pues la misma ha estado sujeta al momento histórico en que se defina y 
a la postura desde donde se aborde; sin embargo, podríamos abordar el concepto como una respuesta emocional positiva hacia el puesto de trabajo (Locke, 1976, 1984). Dicha respuesta es el resultado de la percepción respecto a las condiciones de trabajo.

Durante primera mitad del siglo XIX, la satisfacción laboral fue de interés por su asociación a la reducción de accidentes y demás circunstancias que podrían frenar la producción de las empresas. Sin embargo, tal como lo plantean Chiang, Martín y Nuñez (2010), actualmente esta variable se relaciona directamente con la calidad de vida de los miembros de la empresa, suponiendo una perspectiva más holística del fenómeno, en donde confluyen factores de diversa naturaleza, tanto individuales como colectivos e higiénicos. Así, pues, entrarían a jugar un papel sobre la satisfacción laboral aspectos como los rasgos de personalidad de cada empleado, las formas de relación de la organización, como su estructura jerárquica e incluso las herramientas de trabajo y las condiciones físicas en que el mismo se desarrolle.

Esta postura más holística, sin embargo, ha generado algunas confusiones y aproximaciones erróneas hacia el concepto de la satisfacción laboral. Por ello, es importante para el psicólogo de las organizaciones evitar caer en la generalización y homologación de conceptos que se suelen asociar a la satisfacción laboral, como bienestar, rendimiento, desempeño y clima organizacional; en particular, sobre este último. Salgado, Remeseiro e Iglesias (1996) concluyen, en una investigación, que ni siquiera debería tener relación, pues es posible encontrar organizaciones en donde se presente un buen clima laboral, pero no así satisfacción laboral.

A propósito, Arnold et al. (2012) destacan que la satisfacción laboral se encuentra afectada por aspectos como las actitudes hacia el sueldo, las condiciones de trabajo, por las relaciones con los colegas y con los jefes, las posibilidades de hacer carrera en la empresa y otras cuestiones intrínsecas del trabajo. Por otra parte, Zayas, Báez, Zayas y Hernández (2015, p. 37) plantean que "La satisfacción laboral es un continuo, que va desde de la satisfacción hasta la insatisfacción y no un fenómeno separado". Tal afirmación supone, entonces, que el concepto es dinámico y que el nivel de satisfacción está influenciado por otras variables que deberían tenerse en cuenta para abordar el concepto de satisfacción laboral. 
Una de las variables a tener en cuenta para abordar el concepto de satisfacción laboral es la motivación de la persona, preferiblemente la motivación intrínseca, relacionada con su logro personal, cuando el trabajador realiza su trabajo por placer y sin ningún incentivo externo, sintiendo una sensación de éxito y deseo personal de hacerlo (Carmona, Estellés, Díaz, \& Aguilera, 2018), sin desconocer la importancia de la motivación extrínseca, relacionada con la recompensa y el castigo, más asociada a la teoría operante o instrumental propuesta por Skinner.

Las características de personalidad inciden significativamente sobre la percepción respecto a la satisfacción con la vida y la satisfacción laboral, determinando aspectos como la resiliencia, la frustración y la motivación. De acuerdo con Cornejo (2018), es un constructo teórico que se relaciona con las estrategias conductuales, cognitivas y afectivas que representan procesos mediadores en el organismo, e influencian su respuesta ante escenarios sociales. En este sentido, es posible que una persona con altos rasgos de introversión pueda sentirse incómoda en una labor de alta demanda social. En tal caso, su rasgo de personalidad influenciará negativamente sus posibilidades de sentirse satisfecho en el trabajo que realiza. De acuerdo con Díaz y Sánchez (2002, p. 100) "las características de personalidad modulan las relaciones en la satisfacción de diferentes áreas vitales, siendo significativo el número y tipo de áreas relacionadas, lo que indica que la representación de la satisfacción es diferente según las características personales". Por esta razón, es posible encontrar situaciones donde, a pesar de un entorno laboral que promueva bienestar a la mayoría de las personas, algunas de ellas podrán encontrar el mismo entorno como una amenaza para su propio bienestar y, por supuesto, para su satisfacción laboral.

La satisfacción laboral de las personas también se ve influenciada por aspectos circunstanciales; el contexto social, político y económico en el que se desenvuelve la organización para la cual trabaja puede afectar sus condiciones de bienestar. Es decir, el contexto macrosocial de la organización en el que se desarrolla la organización influye significativamente sobre el bienestar de los empleados (Boria-Reverter, Crespi-Vallbona, \& MascarillaMiró, 2012). Así, por ejemplo, en Colombia, un empleado con contrato a término podría percibir mayor estabilidad en su trabajo que otro cuyo contrato sea por prestación de servicios o por obra o labor. Por otra parte, 
los entornos de trabajo con condiciones precarias de comodidad también impactarán la satisfacción de la persona con su trabajo.

Finalmente, es posible considerar que la satisfacción laboral que una persona perciba respecto a su trabajo es necesaria, no solo para ella, en la medida que le aporta bienestar, sino también para la empresa, pues existen abundantes estudios que evidencian los beneficios que para la empresa implica un empleado satisfecho.

\section{Bienestar psicológico, subjetivo y social}

Un empleado satisfecho gozará de un mayor nivel de bienestar en su dimensión laboral, y tendrá mayor cantidad de recursos cognitivos para mejorar sus condiciones de bienestar en otros aspectos de su vida. Por esto, un gran aporte de estudiar la satisfacción laboral, es que necesariamente quien asuma este reto se topará con el bienestar, adquiriendo posibilidades de hacer una labor de mayor impacto social y empresarial.

Al referirse al bienestar psicológico, subjetivo y social en las organizaciones, es importante esclarecer que estos términos provienen de la psicología positiva, una perspectiva de la psicología que trasciende al tradicional abordaje de la misma desde una perspectiva clínica y asociada a la enfermedad. De hecho, Seligman (2002) propone que la psicología positiva se basa en tres pilares: el estudio de la emoción positiva, el estudio de los rasgos positivos y el estudio de las instituciones positivas.

Al enmarcar a la psicología positiva en el contexto de las organizaciones, se estaría asumiendo, entonces, el potencial de estas para generar y aumentar el bienestar en las personas que las conforman. Pero ello implica también que estas mismas podrían reducir dicho bienestar, generando consecuencias relacionadas con la disminución de la motivación, de la satisfacción laboral e incluso del desempeño del empleado.

Ahora bien, respecto al bienestar psicológico se puede afirmar que se relaciona con la manera en que las personas asumen sus retos vitales, su empeño por alcanzar sus propias metas y afrontar las circunstancias que ello implica. De acuerdo con Freire, Ferradás, Núñez y Valle (2017), alude a la adquisición de los valores que posibilitan la autorrealización. Por esta razón están relacionados con la necesidad de logro de las personas; en este sentido, un empleado con bajo nivel de bienestar psicológico podría 
tener dificultades para alcanzar las metas corporativas que le demande la organización. En particular, porque el bienestar psicológico está relacionado con la capacidad de afrontamiento que tienen las personas; así lo evidencian Romero, Brustad y García (2007) al encontrar la existencia de relación significativa intra-género entre el bienestar y el afrontamiento.

Otro aspecto de interés para un psicólogo en las organizaciones, es conocer el nivel de bienestar subjetivo con el que cuentan los miembros de la organización, es decir, la propia valoración de "felicidad" que poseen los miembros de la organización. Díaz-Llanes (2001) considera que el bienestar psicológico incluye una dimensión cognitiva que se relaciona con la satisfacción con la vida en general, y componentes específicos como el ámbito laboral, familiar y relaciones de pareja entre otros. Y otra dimensión afectiva, relacionada con la frecuencia de emociones positivas y negativas. La interacción de estas emociones determina el bienestar psicológico de la persona.

Ahora bien, es evidente que el estado de bienestar subjetivo de un empleado no depende exclusivamente de factores asociados a la organización; aspectos familiares, el estado de salud, la historia de vida e incluso las características de las relaciones de pareja hacen parte integral de esta percepción. De hecho, en Europa existen políticas de conciliación de la vida familiar y laboral, que parten del reconocimiento de esta situación (Poza, 2010).

Por ello, está en el haber del psicólogo identificar los aspectos asociados a la empresa y fuera de ella— que influyen positiva o negativamente sobre el bienestar psicológico de los miembros de la organización, pero que, aun así, no vulneren su derecho a la privacidad.

Lo anterior permite identificar la importancia de la realización de actividades de gestión humana encaminadas a fortalecer dimensiones personales de los empleados, pues, a pesar de la existencia de condiciones asociadas a la empresa que generan vulnerabilidad en sus empleados, ellos también cuentan con aspectos de tipo personal, con los que ya vienen a las empresas, pero sobre los que posiblemente requieran apoyo. No sirve de mucho hacer grandes esfuerzos en garantizar el bienestar de los empleados en la empresa si solo se asume a la persona como empleado, desconociendo las demás dimensiones que tiene y que, seguramente, explican su bienestar, 
sus emociones, su satisfacción laboral y su desempeño en la organización (Poza, 2010).

Otro tipo de bienestar, relevante para el psicólogo de las organizaciones, es el bienestar social, pues toda organización es en principio un constructo social con una estructura de poder y de roles presumiblemente definidos. Es necesario tener en cuenta lo individual y lo social, el mundo dado y el mundo intersubjetivamente construido Blanco y Díaz (2005). Por esta razón, no sería posible considerar cualquier análisis de bienestar o de vulnerabilidad, si este no está enmarcado en el contexto social de la organización, y por ende en sus grupos o equipos de trabajo.

A diferencia del bienestar psicológico y el bienestar subjetivo, el bienestar social se puede identificar de una manera más objetiva, pues se evidencia en la construcción de relaciones objetivas con los otros (Actis Di Pascuale, 2008). Por tanto, un bajo nivel de bienestar social podría generar conflictos interpresonales e intergrupales en la organización, como también conflictos de tipo estructural.

Podría considerarse, entonces, que los tipos de bienestar mencionados —el psicológico, el subjetivo y el social—, tienen en común que se se forman a partir de la manera en que las personas se experimentan a sí mismas, con su historia y su presente, pero esto implica siempre una contrastación con lo que le refuerce su entorno social y, como resultado, podrán hallarse personas con un cierto nivel de vulnerabilidad ante los entornos de la organización, y, aun, organizaciones vulnerables en sí mismas.

No queda más que recordar, entonces, la importante labor que tiene el psicólogo de las organizaciones en atender los tipos de bienestar expuestos, dado que no son aspectos relacionados solo con el bienestar de las personas, sino con los indicadores de desempeño de la organización, y, más importante, con el valor que esta podría aportar en el desarrollo personal de sus miembros.

\section{Estrategias de prevención y promoción de la salud en el trabajo y las organizaciones}

A partir de lo expuesto hasta ahora, surge entonces la pregunta: ¿Cuáles estrategias puede implementar un psicólogo de las organizaciones para evitar o reducir los factores asociados a la vulnerabilidad de los 
empleados y de la organización misma? Al respecto, podría referenciarse cientos de métodos; sin embargo, por efectos prácticos, se considerarán solo algunos que generan impactos importantes sobre los tipos de bienestar abordados y sobre la satisfacción laboral de los empleados.

En primer lugar, es necesario que toda organización cuente con una estructura evidenciada en un organigrama. Esto no solo le permite a cada empleado ubicarse en la organización, sino también conocer el alcance de su rol y las líneas de poder dentro de la estructura; podrá saber a quién reportar alguna novedad y con quién no debería hacerlo. Adicional a esto, le permite saber que es parte de unos procesos específicos de la organización, evitando el estar desubicado y siguiendo, quizás, órdenes de quien no debe, o haciendo funciones que no le corresponden y por las cuales no será recompensado.

El contar con una estructura en la organización lleva a una segunda estrategia para reducir los factores asociados a la vulnerabilidad, y es la creación de diseños de puestos de trabajo, pues en estos se establecen los parámetros básicos sobre los cuales cada empleado desarrolla una labor dentro de la organización. Para Wall (1982), un diseño de puestos de trabajo permite incrementar los siguientes aspectos: variedad de las tareas o destrezas; autonomía, que se refiere a la libertad para elegir métodos de trabajo; y, por último, la integración, que es el grado en el que el trabajo genera un resultado final identificable al que el individuo puede apuntar. En este sentido el diseño del trabajo debería indicar las tareas asociadas al cargo, las características de la persona idónea para el cargo y los requerimientos mínimos de equipos con los que debería contar la persona para hacer su trabajo adecuadamente.

Los diseños de puestos de trabajo también son de gran utilidad para los procesos de selección, pues le evitan al psicólogo de la organización contratar personas que no se ajusten a las características del cargo, bien sea por aspectos de tipo personal, por carencia de las competencias asociadas a la tarea o por cualquier parámetro que no se ajuste al diseño del puesto.

Formar a los líderes de la organización, sobre modelos adecuados de dirección, también es importante; un estilo de dirección Laissez-faire puede generar desmotivación en los empleados, pues pueden sentir que su trabajo no importa o simplemente no sienten interés de hacer las cosas de mejor 
manera, podría dar igual en la medida en que, difícilmente, tendrán una devolución o feedback de su trabajo.

Por otra parte, un liderazgo autocrático puede generar malestar entre los empleados, en la medida en que sus opiniones no son tenidas en cuenta y, en algunos casos, el líder autocrático puede obviar algunos derechos de los empleados, lo que podría ser percibido como acoso laboral. Por tal motivo, un liderazgo transformacional, es donde el líder inspira a sus seguidores para que trasciendan sus intereses personales y, además, genera efectos profundos sobre ellos (Robbins y Judge, 2017). Este tipo de liderazgo podría ser el mejor escenario para evitar la percepción de vulnerabilidad entre los empleados de la organización.

Las actividades de promoción, en conductas saludables y prevención de enfermedades laborales, también incrementan el sentido de pertenencia de los empleados, en la medida en que pueden sentirse valorados, no sólo como empleados, sino como personas. Estas actividades, usualmente, son de bajo costo, pero de gran impacto; incluso la empresa tiene derecho, de forma gratuita, a algunas de ellas, pues al afiliar a sus empleados a la Aseguradora de Riesgos Laborales (ARL) tiene derecho, sin costo adicional, a actividades de prevención de enfermedades laborales.

Podríamos continuar enumerando alternativas para reducir la vulnerabilidad de los empleados; sin embargo, es importante tener en cuenta que cada alternativa utilizada deberá desprenderse de un diagnóstico inicial que el psicólogo realice sobre la organización. Es este diagnóstico el que le indicará cuál estrategia generará un mayor impacto sobre la reducción de los índices de vulnerabilidad. En este sentido, no importa el número de estrategias empleadas; importa la pertinencia de las mismas con la situación de los empleados en la organización.

\section{Conclusiones}

En este capítulo se ha abordado el tema de la vulnerabilidad en el mundo laboral, entendiéndolo — desde la psicología del trabajo y de las organizaciones - como aquellos factores que ponen en riesgo la salud física y mental de los trabajadores. Para la comprensión de dicha vulnerabilidad se trabajaron los diferentes factores de riesgo, como la carga laboral, el 
contenido de la tarea, los horarios de trabajo, el estrés, el acoso laboral, entre otros. De igual manera, se mencionaron algunas investigaciones que le permiten al lector entender — de forma muy general— la afección de estos factores en la salud del trabajador.

En la misma línea, se trabajó el concepto de salud en el trabajo desde una perspectiva positiva de la psicología, que comprende la salud mental como el bienestar y no solo como la ausencia de enfermedad. De esta manera, la salud implica un estado de bienestar completo en el que subyacen un grupo de dimensiones subjetivas, psicológicas y sociales que pueden verse afectadas o protegidas en el campo laboral. Asimismo, se tuvieron en cuenta elementos como la calidad de vida en el trabajo y la satisfacción laboral, que se encuentran relacionados con la salud de las personas y el éxito de las organizaciones.

Finalmente, se abordaron algunas estrategias que se implementan regularmente en las organizaciones para facilitar la salud y la seguridad de los trabajadores, como lo relacionado con la estructura organizacional, el diseño del trabajo, la promoción de las conductas saludables, entre otras. Se hace énfasis en la importancia de realizar diagnósticos organizacionales que permitan comprender mejor la realidad y las problemáticas de la organización, para definir, de manera alineada, las estrategias de intervención y,x así, asegurar la salud de los trabajadores y de la organización.

\section{Referencias}

Actis Di Pascuale, E. (22-23 de septiembre de 2008). El bienestar social: su concepto y medición de la dinámica histórica en la Argentina. XXI JORNADAS DE HISTORIA ECONÓMICA. Buenos Aires, Caseros, Argentina. Recuperado el 6 de junio de 2018, de http://nulan.mdp. edu.ar/808/1/00474.pdf

Arnold, J., Randall, R., Patterson, F., Silvester, J., Robertson, I., Cooper C., Burnes, B., Swailes, S., Harris, D., Axtell, C., \& Den Hartoget, D. (2012). Psicología del Trabajo. Comportamiento humano en el ámbito laboral. México: Pearson Educación.

Blanco, A., \& Díaz, D. (2005). El bienestar social: su concepto y medición. Psicothema, 17(4), 582-589. Recuperado el 6 de junio de 2018, de http://www.psicothema.es/pdf/3149.pdf 
Bond, F. W., Flaxman, P. E., \& Loivette, S. (2006). A business case for the Management Standards for stress. Health and Safety Executive (Research Report 431). Recuperado de http://www.hse.gov.uk/research/rrpdf/rr431.pdf

Boria-Reverter, S., Crespi-Vallona, M., \& Mascarilla-Miró, O. (2012). Variables determinantes de la satisfacción laboral en España. Cuadernos de economía, 35, 9-16. https://doi.org/10.1016/S02100266(12)70018-3

Buden, J. C., Dugan, A. G., Namazi, S., Huedo-Medina, T. B., Cherniack, M. G., \& Faghri, P. D. (2016). Work Characteristics as Predictors of Correctional Supervisors' Health Outcomes. Journal of Occupational and Environmental Medicine, 58(9), e325-e334. https://doi. org/10.1097/JOM.0000000000000843

Camargo, J., \& Puentes, A. (2010). Rasgos de personalidad y autoestima en víctimas de acoso laboral. Revista Diversitas - Pespectivas en Psicología, 6(1), 51-64. Recuperado de https://www.redalyc.org/articulo. oa?id=67916261005

Carmona, E., Estellés, I., Díaz, G., \& Aguilera, R. (2018). Nivel de satisfacción y compromiso en trabajadores remunerados versus voluntariado y motivación en el mismo. ReiDoCrea, 7, 85-91. https://www. ugr.es/ reidocrea/7-8.pdf

Chiang, M., Martín, M., \& Nuñez, A. (2010). Relaciones entre el clima organizacional y la satisfacción laboral. Madrid: Universidad Pontifica Comillas.

Congreso de la República de Colombia. (2006). Ley 1010 de 2006 Por medio de la cual se adoptan medidas para prevenir, corregir y sancionar el acoso laboral y otros hostigamientos en el marco de las relaciones de trabajo. Diario Oficial No. 46.160, de 23 de enero de 2006.

Cornejo-Yarquelé, J. (2018). Temperamentos de personalidad según el modelo de Eysenck y nivel de síndrome de burnout en personal de tropa hospitalizados en el Hospital Militar Central "Coronel Luis Arias Schreiber" (Trabajo de Pregrado) Universidad Inca Garcilaso De La Vega, Lima, Perú. Recuperado de http://repositorio.uigv.edu.pe/ bitstream/handle/20.500.11818/2156/TESIS_Jorge\%20Luis\%20 Cornejo\%20Yarlequ\%C3\%A9.pdf? sequence=2 ErisAllowed $=y$ 
Díaz-Llanes, G. (2001). El bienestar subjetivo. Actualidad y perspectivas. Revista Cubana de Medicina General Integral, 17(6), 572-579. Recuperado el 6 de Junio de 2018, de http://scielo.sld.cu/scielo. php?script=sci_arttext\&pid=S0864-21252001000600011\&ln$\mathrm{g}=\mathrm{es} \& \mathrm{t} \operatorname{lng}=\mathrm{es}$.

Díaz-Morales, J. F., \& Sánchez-López, M. P. (2002). Relaciones entre estilos de personalidad y satisfacción autopercibida en diferentes áreas vitales. Psicothema, 14(1), 100-105. Recuperado de http://www. psicothema.com/pdf/692.pdf

Donders, N. C. G. M., Roskes, K., \& Van der Gulden, J. W. J. (2007). Fatigue, emotional exhaustion and perceived health complaints associated with work-related characteristics in employees with and without chronic diseases. International Archives of Occupational and Environmental Health, 80(7), 577-587. https://doi.org/10.1007/ s00420-006-0168-6

Fernández-Montalvo, J., \& Pinol, E. (2000). Horario Laboral Y Salud: Consecuencias Psicológicas De Los Turnos De Trabajo. Revista de Psicopatología y Psicología Clínica, 3(5), 207-222. Recuperado de http://www.aepcp.net/arc/04.2000(3).Fernandez-Pi\%C3\%Blol.pdf

Freire, C., Ferradás, M., Núñez, J., \& Valle, A. (2017). Estructura factorial de las Escalas de Bienestar Psicológico de Ryff en estudiantes universitarios. European Journal of Education and Psychology, 10, 1-8. https://doi.org/10.1016/j.ejeps.2016.10.001

Gómez, I. (2009). "Promoción de la salud en el lugar de trabajo". En Psicología del trabajo y las organizaciones. Reflexiones y experiencias de investigación. M. C. Aguilar \& E. Rentería (Editores) Bogotá: Universidad Santo Tomas y Universidad del Valle.

González-Muños, E., \& Gutiérrez-Martínez, R. E. (2006). 2_Factor De Riesgo EnIndustriaElectronica. RevistaLatinoamericanadePsicología, 38(2), 259-270. Recuperado de http://www.scielo.org.co/scielo.php?script=sci_arttext\&pid=S0120-05342006000200003\&lng=en\&nr$\mathrm{m}=\mathrm{iso}$

Kamp, J. (1994). Worker safety: psychology management's next frontier. Professional Safety, 39(5), 32-33.

Keyes, C. L. (2005). Mental illness and/or mental health? Investigating axioms of the complete state model of health. Journal of Con- 
sulting and Clinical Psychology, 73, 539-548. http://dx.doi.org/10.1037/0022-006X.73.3.539

Leka S., \& Cox T., Eds. (2009). PRIMA-EF: guidance on the European framework for psychosocial risk management: a resource for employers and worker representatives. Protecting Workers' Health Series \#9. World Health Organization. Recuperado de http://www.who. int/occupational_health/publications/PRIMA-EF\%20Guidance_9. pdf

Locke, E. A. (1976). The nature and causes of job satisfaction. En M.D. Dunnette (ed.) Handbook of Industrial and Organizational Psychology. Chicago: Rand McNally.

Locke, E. A. (1984). Job satisfaction. En M. Gruneberg \& T. Wall (eds.) Social Psychology and Organizational Behaviour. Chichester: Wiley

Morgeson, F. P., \& Campion, M. A. (2002). Minimizing tradeoffs when redesigning work: Evidence from a longitudinal quasi-experiment. Personnel Psychology, 55(3), 589-612. https://doi.org/10.1111/j.1744-6570.2002.tb00122.x

Muñoz-Chávez, J., López-Chau, A., \& González-Pérez, M. (2018). Estudio Sobre La Relación Entre Mobbing Y La Satisfacción Laboral En Docentes De Instituciones De Educación Superior En México. European Scientific Journal, 14, 298-314. http://dx.doi.org/10.19044/ esj.2018.v14n4p298

Organización Mundial de la Salud [OMS] (2010). Entornos Laborales Saludables: Fundamentos y Modelo de la OMS. Contextualización, Prácticas y Literatura de Apoyo. Ediciones de la OMS. Ginebra Suiza. Recuperado de https://www.who.int/occupational_health/ evelyn_hwp_spanish.pdf

Palumbo, A., De Roos, A., Cannuscio, C., Robinson, L., Mossey, J., Weitlauf, J., ... \& Michael, Y. (2017). Work Characteristics Associated with Physical Functioning in Women. International Journal of Environmental Research and Public Health, 14(4), 424. https://doi. org/10.3390/ijerph14040424

Park, R., \& Searcy, D. (2012). Job Autonomy as a Predictor of Mental Well-Being: The Moderating Role of Quality-Competitive Environment. Journal of Business and Psychology, 27(3), 305-316. https:// doi.org/10.1007/s10869-011-9244-3 
Peralta, M. (2004). El acoso laboral - mobbing- Perspectiva psicológica. Revista de Estudios Sociales, 18, 111-122. Recuperado de http://www.scielo.org.co/scielo.php?script=sci_arttext\&pi$\mathrm{d}=$ S0123-885X2004000200012

Polo-Vargas, J. (2012). Diseño del trabajo y bienestar. Un aporte dese la psicología organizacional positiva. (Tesis doctoral) Universidad Autónoma De Madrid, Madrid. Recuperado de https://repositorio. uam.es/xmlui/bitstream/handle/10486/11179/55834_polo_vargas_jean_david.pdf?sequence $=1$ \&isAllowed $=y$

Polo-Vargas, J., Fernández-Ríos, M., \& Ramírez-Vielma, R. (2012). Diseño del trabajo y satisfacción con la vida. Revista Venezolana de Gerencia, 17(59), 466. Recuperado de https://www.redalyc.org/articulo. oa?id=29024166005

Poza Campillo, I. (2010). Políticas de conciliación de la vida laboral y familiar en los regímenes de bienestar mediterráneos: los casos de Italia y España. Política y Sociedad, 47(1), 189-213. Recuperado de http:// revistas.ucm.es/index.php/POSO/article/view/POSO1010130189A

Romero-Carrasco, A., Brustad, R., \& García-Mas, A. (2007). Bienestar psicológico y su uso en la psicología del ejercicio, la actividad física y el deporte. Revista de iberoamericana de psicología del ejercicio y el deporte, 2(2), 31-52. Recuperado de https://www.redalyc.org/ articulo.oa?id $=311126258003$

Rodríguez-López, A.I., \& Méndez-Durán, A. (2016). Síndrome de burnout en profesionales de áreas críticas en un hospital de tercer nivel. Gaceta médica de Bilbao, 113(3), 99-104. Recuperado de http:// www.gacetamedicabilbao.eus/index.php/gacetamedicabilbao/article/view/120

Robbins, S.P., \& Judge, T.A. (2017). Comportamiento Organizacional (17 ed.). Ciudad de México, México: Pearson.

Rydstedt, L., Johansson, G., \& Evans, G. W. (1998). A longitudinal study of workload, health and well-being among male and female urban bus drivers. Journal of Occupational, 71(1), 35-45. https://doi.org/10.1111/j.2044-8325.1998.tb00661.x

Salgado, J., Remeseiro-Rioboo, C., \& Iglesias, M. (1996). Clima organizacional y satisfacción laboral en una PYME. Psicothema, 8(2), 329335. Recuperado de http://www.psicothema.com/pdf/31.pdf 
Sarango Molina, W. A. (2017). Sistematización de la experiencia del diseño y mejoramiento del manual de funciones y perfiles de cargo del área de talento humano, en una institución del estado, durante el periodo marzo - agosto 2016. (Tesis de pregrado) Universidad Politécnica Salesiana, Quito.

Seligman, M. E. P. (2002). Authentic happiness: Using the new positive psychology to realize your potential for lasting fulfillment. NewYork: Free Press

Topa, G., Depolo, M., \& Morales, F. (2007). Acoso laboral: meta-análisis y modelo integrador de sus antecedentes y consecuencias. Psicothema, 19(1), 88-94. Recuperado de https://www.redalyc.org/articulo. oa? id $=72719113$

Wall, T. D. (1982) "Perspectives on job redesign", en J.E. Kelly \& C.W. Clegg (eds.), Autonomy and Control in the Workplace. Londres: Croom Helm.

Zayas-Agüero, P.M.; Báez-Santana, R.A., Zayas-Feria, J., \& Hernández-Lobaina, M. (2015). Causas de la satisfacción laboral en una organización comercializadora mayorista. Revista Facultad de Ciencias Económicas, 35-51. http://dx.doi.org/10.18359/rfce.1606 